\title{
Management of Biceps Brachii Tendon Disorders
}

\author{
M.A.Mashhour, E.M.Ibrahim, E.M.Bayomy and A.M.Abd El Hamid
}

Orthopedic Surgery Dept., Faculty of Medicine, Benha Univ., Benha, Egypt

E-Mail: gifo23@yahoo.com

\begin{abstract}
The Long Head of Biceps originates at the supraglenoid tubercle and the superior glenoid labrum. It inserts distally, along with the short head of the biceps, onto the radial tuberosity, with an attachment to the fascia of the medial forearm via the bicipital aponeurosis. The site of the LHB origin from the glenoid labrum is variable; in most cases, it arises either mostly posterior or completely posterior [55.4\% and $27.7 \%$, respectively]. Type I lesions are treated with conservative debridement of the frayed labrum. Type II lesions are repaired using a single-anchor, double-suture technique. Type III lesions are treated by initial resection of the unstable bucket-handle labral fragment, followed by inspection of the biceps anchor attachment for stability. Type IV lesions are treated as type III lesions unless the biceps tendon split is severe. When more than approximately $30 \%$ of the tendon is included with the displaced labral tear one must consider either repairing the tendon, or releasing it and repairing the labrum as with a type II SLAP, or performing a biceps tenodesis . the decision depends on the age and activity level of the patient and the condition of the remainder of the biceps tendon.
\end{abstract}

\section{Introduction}

The biceps muscle has two separate heads. The short head and the coracobrachialis originate from the coracoid process and combine to form the conjoined tendon. The long head originates within the glenohumeral joint and, after coursing through the bicipital groove between the greater and lesser tuberosities, joins with the short head at the level of the deltoid insertion to form the muscle proper [1].

\section{Anatomy}

\subsection{Gross anatomy}

[2] studied 100 cadaveric shoulders with histologic sectioning and noted that in each specimen, 40 to $60 \%$ of the fibers of the long head of the biceps tendon arose from the superior glenoid labrum, with the remainder originating from the supraglenoid tubercle.The long head of the biceps tendon is intimately associated with the superior aspect of the glenoid labrum, separated only by a synovial reflection [2].

Recommended caution in assessing the superior labrum at the biceps origin, suggesting that some looseness and mobility of the labrum in this region may be normal, and that debridement may not be indicated as treatment for a presumed SLAP lesion.

\subsection{Arthroscopic anatomy}

Successful shoulder arthroscopy depends upon a systematic approach and thorough knowledge of the anatomy of the glenohumeral joint. Variations in the normal anatomy are uncommon. To achieve a thorough and reproducible arthroscopic examination, structures should be identified and examined systematically. The biceps tendon is identified first since it is used as the main reference point throughout the whole arthroscopic examination [3].

\section{Biomechanics of the Biceps 3.1 Tendon}

The shoulder has more freedom of movement than any other joint in the body. The large range of motion and the relatively shallow configuration of the joint predispose the shoulder to injury. The soft tissue structures around the joint play a vital role in the stability of the shoulder. Normally, the stability of the glenohumeral joint is maintained by complex interactions of the muscles and ligaments that cross the joint. No single structure is responsible for stability of the joint in all positions of the

A biomechanical study by [4] demonstrated that application of a 3-kg load to the long head of the biceps resulted in decreased anterior humeral head translation with the shoulder in $90^{\circ}$ of abduction and $60^{\circ}$ to $90^{\circ}$ of external rotation [4].

Dynamic depression of the humeral head was demonstrated by [9] who showed superior migration of the humeral head relative to the contra lateral [control] shoulder in seven patients with isolated loss of the proximal attachment of the long head of the biceps. However, it has been demonstrated that no significant superior migration of the humeral head occurs at all with arthroscopic release of the biceps tendon in the presence of irreparable massive rotator cuff tears [5].

[6] applied forces to the biceps tendon in 10 cadavers and found significantly less translation of the humeral head in the superior, anterior, and inferior directions when these forces were applied [6].

\subsection{The effect of cutting of biceps origin}

[7] tested the origin of the long head of the biceps tendon under tension by selectively cutting its two origins [supraglenoid tubercle and superior labrum] to determine each of their contributions to stiffness and maximum displacement under a given load. In this model, the biceps anchor at the supraglenoid tubercle was consistently shown to function as the primary restraint under tension 
applied perpendicular to the face of the glenoid. The superior labral attachment was the secondary restraint under these conditions [7].

\section{Types of bicepes tendon lesions}

Multiple classification schemes have been developed to describe disorders of the Long head of biceps tendon. These divisions have been only marginally useful in regards to diagnosis and treatment decisions. More important is to understand the various pathological processes involving the biceps tendon and how to treat each process accordingly. The three biceps tendon pathologies that will be discussed are biceps tendinitis, rupture, and instability. Lesions involving the biceps origin [SLAP lesions] will be discussed in greater detail [8].

\subsection{Classification of biceps tendon disorders}

\subsubsection{Slatis and Aalto classification}

\subsubsection{Type 1 Impingement tendinitis}

This type is secondary to impingement syndrome and rotator cuff disease. The torn cuff exposes the biceps tendon to the rigid coracoacromial arch and results in tendinitis. This is the most frequent cause of biceps tendinitis [9].

\subsubsection{Type 2 Subluxation of the biceps tendon}

This lesion can occur in an isolated fashion or it can be associated with tears of the supraspinatus and the subscapularis. As the tendon slips in an out of its sulcus, it develops inflammation and fraying. The tendon can finally fully displace into a sling ruptured cuff in early cases. In more severe and later cases, the tendon may actually dislocate intraarticularly. The groove becomes shallower during this process as it fills with scar tissue [9].

\subsubsection{Type 3 Attrition tendinitis}

These lesions are primary lesions of the biceps tendon that occur inside the canal. In this type of tendinitis, inflammation in the tight canal causes pain and degeneration of the biceps tendon. These changes are associated with spurring and fraying of the tendon. Inflamation of the sheath causes formation of spurs and stenosis of the groove. This condition is though to be extremely painful and rare [9].

\subsubsection{Habermeyer and Walch's classification}

\subsubsection{Origin lesions}

These are lesions affecting the attachment of the biceps tendon to the supraglenoid tubercle and to the superior glenoid labrum. These lesions are usually due to traction injuries in throwing athletes, especially during the phase of throwing. These lesions can also be caused by falls on the outstretched arm with the shoulder in abduction and slight forward flexion. Acute inferior traction as well as abduction-external rotation injuries can also cause this lesion [10] .

\subsubsection{Interval lesions}

\subsection{Biceps tendinitis}

Biceps tendinitis is clinically characterized by chronic shoulder pain with tenderness over the bicepital groove and a positive speed's test. When these criteria are used, $90 \%$ of all painful shoulders could be considered as having biceps tendonitis. However, on pathologic examination biceps tendon in these shoulders rarely shows degeneration or microtraumatic lesions. These changes have been reported in only $5 \%$ of cases [10].

\subsubsection{Biceps tendon disorders associated with rotator cuff tears}

4.1.3.1 Biceps tendinitis associated with cuff tears

In this subgroup, patients have tendinitis of the biceps secondary to exposure of the biceps to the rigid coracoacromial arch. These patients have a rotator cuff tear but no dislocation or subluxation of the biceps tendon. The biceps is inflamed and painful and may appear hypertrophied when viewed through the arthroscope [10].

\subsubsection{Ruptures of the biceps tendon associated with cuff tears}

The biceps can also rupture due to rotator cuff tears. This is usually due to impingement of the biceps and supraspinatus tendons in the area of the biceps sulcus. has shown that these lesions are frequently associated. Most ruptures of the biceps tendon are associated with rotator cuff tears, while isolated ruptures are rare [11].

Chronic biceps tendinitis is a more common etiology resulting in eventual Long head of biceps rupture. The Long head of biceps becomes attenuated and weakened by the continued impingement between the humeral head and the coracoacromial arch. In these cases of impingement causing rupture, the rupture typically occurs around the area of the rotator cuff interval [a weak point for the Long head of biceps] rather than at its origin [12].

\section{Diagnosis of biceps tendon lesions}

The evaluation and treatment of shoulder pathology has been a challenge for orthopedic surgeons. Due to its complex anatomy and the difficulty of direct examination the role of the biceps tendon as a source of shoulder pain has received much attention in the orthopedic literature. With advances in imaging techniques and arthroscopy, the controversy over the role of the biceps tendon as a source of shoulder pathology can be settled by a thorough evaluation, 
which permits treatment to be tailored directly to the needs of the patient [13].

\subsection{Clinical evaluation \\ 5.1.1 History}

Although rare, there may be a history of previous injury to the proximal shoulder that could cause an alteration in the anatomy of the groove [13].

The patient with an acute rupture of the long head of the biceps frequently has a long history of shoulder pain, which was actually relieved somewhat after the tendon ruptured, and complains of deformity and soreness more distally in the arm [13].

\subsubsection{Physical examination}

Passive and active range of motion of the shoulder should be measured in both the standing and the supine positions. There is slight limitation of abduction and internal rotation. This loss of motion is usually due to pain and should improve with local anesthetic injection [12].

\subsubsection{Arthroscopy}

\subsection{Bicieps tendon disorders}

However, as the extra-articular portion of the tendon may also have pathologic changes, it should not be overlooked. This region of the tendon may be readily inspected with one easy maneuver. Through an anterior portal, a probe is used to push the tendon inferiorly to determine the pliability and mobility of the tendon. By pulling the intertubercular portion of the tendon back into the intra-articular space, an additional 3 to $5 \mathrm{~cm}$ of the tendon can be inspected [Fig.2-31]. In some instances, fraying or degeneration of this portion of the tendon is an occult source of shoulder pain [18].

Arthroscopy is also an excellent method for evaluating the biceps anchor region and for documenting avulsion tears of the tendon and superior labrum anteroposterior [SLAP] lesions [14].

\subsection{Rotator cuff lesions}

However, by arthroscopic inspection alone, differentiation between a small full thickness tear and a superficial tear could be difficult. Palpation of the cuff with a probe made this easier. The cuff could be examined in this way from the inside, or from the outside if the probe was inserted laterally into the subacromial space. To some extent, differentiation between an old and a recent rupture is possible. According to the shape of the rupture edges, which are smooth in an old rupture, and frayed and uneven in a recent rupture [15].

The rotator cuff should be visualized completely on both its articular and bursal sides. Bursoscopy also permits direct visualization of the undersurface of the acromion to detect evidence of impingement, which can be treated by arthroscopic decompression [14].

Cuff rupture is often combined with degenerative changes of the biceps tendon, mostly in the part of the bicipital groove, or proximal to this part where it lies between the humeral head and the rotator cuff. Partial tear of the biceps tendon is frequently seen with full thickness rupture of the cuff. A slight reactive synovitis is seen in most cases with degenerative changes of the articular surface [16].

\section{Treatment}

6.1 Non surgical treatment of biceps tendon lesions

The treatment of lesions of the biceps tendon, with the exception of acute traumatic rupture of the tendon in the young active patient or in association with a massive cuff tear in an active patient, should begin with prolonged conservative care before any thought of operative intervention [12].

\subsubsection{Corticosteroid-anesthetic injections}

Occasionally, when the symptoms in the acute period are severe or when it becomes imperative for an athlete to return to his or her sport as soon as possible, a small amount of corticosteroid and a local anesthetic agent can be injected into the bicipital area [13].

There should be no resistance to the injection, as resistance implies intratendinous insertion of the needle. Use of a 20-gauge needle for this technique allows ready detection of flow resistance. After 5 minutes, the patient is reexamined, and any changes are noted [5].

The specific increase in the risk of rupturing the degenerative biceps tendon after such an injection is unclear. However, the danger to tendinous structures following steroid injection is well known. It is critical that the patient be informed of the risk of tendon rupture before injection [5].

\subsubsection{Rehabilitation program}

Because biceps activity increases during follow-through when the external rotators begin to fatigue, these muscles should be specifically targeted during the strengthening program. The internal rotators should also be strengthened, as it is this group that works eccentrically during late cocking to avoid excessive strain within the anterior static restraints. The serratus anterior and trapezius should be strengthened to allow synchronous motion of the scapula with the humerus and to avoid impingement of the biceps due to scapular lag. Finally, rehabilitation of the biceps itself is emphasized, with a focus on eccentric loading. Once strength and endurance in these muscles have been restored, a return to 
overhead activity is allowed. This is done on a graduated basis, slowly progressing to competitive distance, velocity, duration, and frequency of activity [5].

Given sufficient time, most patients will respond to conservative therapy. If symptoms persist despite a well-managed supervised rehabilitation program, operative intervention may be indicated.

\subsection{Surgical treatment of biceps tendon lesions 6.2.1 Surgical treatment}

6.2.1.1 SURGICAL Treatment of Tendinitis, partially ruptured or subluxated biceps tendon

Surgery should be considered if conservative measures fail after three months. Structures causing primary and secondary impingement may be removed, and the biceps tendon may be repaired if necessary. Debridement should be performed if less than 50 percent of the biceps tendon is torn [17].

\subsection{Tendon degeneration}

The proper surgical treatment of biceps tendinopathy rests partly on the question of whether isolated primary degeneration of the biceps occurs [5].

Primary biceps tendinitis can be treated by a solitary biceps tenodesis without accompanying subacromial decompression, especially in the younger patient with no evidence of associated impingement syndrome [13].

If there are accompanying signs and symptoms of impingement syndrome, a complete subacromial decompression is performed. The question then remains whether to perform a biceps tenodesis or a release with resection of the proximal tendon. This is a difficult decision, particularly in the case of a professional or elite thrower in whom the effect of loss of the depressive and stabilizing function of the biceps is unknown [5].

An alternative approach is to perform tenotomy of the biceps tendon without tenodesis, accepting the inherent deformity, which occurs as the long head retracts into the arm. This approach is often reasonable in older patients and in rare instances in younger patients who are not concerned with deformity or who wish a quicker recovery [5].

\subsection{Tendon instability}

In patients with instability of the biceps tendon, conservative care should again start with resting the extremity and discontinuing the sport that caused the snapping or subluxation of the tendon. If conservative care fails, surgical evaluation should include arthroscopy and exploration of the groove, with specific emphasis on discovering associated rotator cuff and coracohumeral ligament tears [13].

\subsection{Tendon rupture}

For ruptures of the biceps tendon in older inactive patients, conservative care consisting of non steroidal agents and rest for 2 to 3 weeks results in only a mild cosmetic deformity and a functional deficit estimated at $7 \%$ to $10 \%$ of available flexion and $17 \%$ to $20 \%$ of supination [18].

\subsubsection{Open surgical techniques}

Several options are available to the orthopaedic surgeon for stabilisation of the biceps tendon in the intertuberculer groove [19].

described soft tissue fixation in the groove. With an osteotome, a bed is made in the intertubercular groove by elevating a portion of the floor from outside inward [osteol periosteal flap]. The tendon is roughened and then sutured beneath this osteal periosteal flap with heavy nonabsorbable sutures

described a technique of bone fixation through a keyhole tenodesis, which permited early rehabilitation [Fig. 50, C]. In the keyhole technique, the free edge of the tendon is knotted and sutured. A burr is used to create a keyhole, wider proximally to accommodate the knotted tendon and narrowing distally. The knotted tendon is inserted into the keyhole under slight tension, locking it into place. The transverse ligament is then sutured over the tendon.

Used a technique that is a modification of those previously described and that permits excellent fixation of the tendon to bone and maintenance of the normal tension of the tendon [14].

\subsubsection{Arthroscopic treatment}

The initial operative evaluation involves an examination under anesthesia. The shoulder is examined to assess passive range of motion, inferior sulcus translation compared with the contralateral side, and presence of asymmetric anterior or posterior load and shift at $0^{\circ}$ and $90^{\circ}$ of forward elevation in the scapular plane. A diagnostic arthroscopy is performed next [20].

\subsection{Diagnostic arthroscopy}

Traction on the biceps tendon will demonstrate any loss of integrity at the labral attachment as well as instability of the attached ligaments. If traction causes the labrum to arch away from the glenoid, revealing a significant gap of $5 \mathrm{~mm}$ or more, the stability of the anchor is questionable. Likewise, if the attached anterosuperior labrum, middle glenohumeral ligament, or Buford complex is tensioned when the biceps is pulled, then the stability of the biceps-labral-ligament complex is likely deficient [21].

$\begin{array}{lll}\text { 6.2.2.1.1.1 } & \text { Operative } & \text { Techniques } \\ \text { Biceps Tenodesis }\end{array}$


Operative intervention for biceps pathology begins with arthroscopic inspection and debridement. The proximal biceps tendon is easily visualized during standard glenohumeral arthroscopy. The tendon is first visualized thoroughly from the posterior portal. The tendon should be inspected from its origin on the superior glenoid tubercle and/or superior labrum all the way into the bicipital sheath. It is examined for fraying, degenerative changes, thickening or synovitis. The tendon from inside the bicipital sheath should be pulled into the joint with a probe to assess the integrity of the tendon. This can be facilitated by flexing the elbow, supinating the forearm, externally rotating and abducting the arm [22].

\subsection{The Bio-Tenodesis Screw System}

After completion of diagnostic arthroscopy, any tendon degeneration is debrided. If a concomitant rotator cuff tear is present, a lateral portal is established directly through the defect created by the torn rotator cuff. Two racking stitches are placed into the biceps tendon [23].

\subsection{Suture Anchor Technique}

A standard posterior portal is used and entering the glenohumeral joint. The biceps tendon is visualized and areas of fraying, inflammation, or partial tear are noted. A probe is introduced anteriorly and traction is placed on the tendon to bring the extra-articular tendon within view. A spinal needle is introduced percutaneously near the anterolateral acromial border and pierces the tendon just proximal to its exit from the joint

\section{Post Operative instructions after a Biceps} Tenodesis

-Day 1: The Day of Surgery: Maintain dressing, adding bandages if needed for drainage through the dressing. Use ice pack for 20 minute periods throughout today. When sleeping, most patients find that sleeping in a semi-upright position in a recliner or propped up on pillows on a couch is much more comfortable than trying to lie in bed for the first few weeks after shoulder surgery.

-Day 2: The Day after Surgery: Same as Day 1

-Day 3: Starting about 48 hours after surgery: Continue same activities, including using ice for 20 minute periods as needed.

-Day 3- 4: Start Therapy with a physical therapist: This is to help avoid a frozen shoulder.

\section{Rehabilitation Protocol after surgical treatment of slap lesions \\ The patient is placed in an Ultrasling} immobilizer for the first 3 weeks. During the first 4 weeks after surgery, the patient begins passive forward elevation, and full elbow range of motion is permitted. Abduction and external rotation are avoided.

During weeks 4 through 6 passive and active range of motion to $90^{\circ}$ of flexion is the goal. Active forward flexion beyond $90^{\circ}$ and forceful active biceps contraction should be avoided for approximately 6 weeks postoperatively. If a posterior-inferior capsulotomy is performed, sleeper stretches are started immediately postoperatively to stretch the posterior capsule.

After the sixth week, full range of motion including abduction and external rotation and gradual strengthening are performed. Posterior rotator cuff strengthening should be emphasized. Overhead activities or strenuous biceps activity should be avoided for 12 weeks.

At 12 to 16 weeks, physical therapy is discontinued and the patient may return to normal activities. Gentle interval throwing is resumed at 4 to 5 months. Return to unrestricted overhead sports is permitted at 8 to 9 months postoperatively [24].

\section{Summary}

The long head of biceps tendon originates within the glenohumeral joint from the supraglenoid tubercle and the glenoid labrum. The tendon is intra-articular but extrasynovial. As the tendon leaves the glenohumeral joint, it passes deep to the coracohumeral ligament and through the rotator interval, entering the bicipital groove

Because of the intimate association of the biceps tendon with the rotator cuff, the principal cause of biceps degeneration is thought to be mechanical irritation of the tendon against the coracoacromial arch. Similarly, in most patients with subluxation or dislocation of the biceps tendon.

Patients with biceps pathology usually describe pain over the anterior aspect of the shoulder, radiating down over the muscle belly of the biceps. The pain is worse with repetitive overhead activities or lifting and is relieved by rest. By examination there is point tenderness in the biceps groove. Several tests are also described to help in diagnosing biceps pathology. In patients with complete biceps rupture there is hollowness in the anterior portion of the shoulder accompanied by balling up of the biceps below the midbrachium "Popeye sign".

The expanded role of arthroscopy in the diagnosis and management of shoulder disorders in general has Improved our understanding of pathologic conditions involving the glenoid labrum and biceps tendon. One group of lesions involves tearing of the superior labrum specifically located in the anterosuperior quadrant of the glenoid near the origin of the long head of the biceps and termed SLAP lesions .

The incidence, the etiology of Biceps tendon lesions remain uncertain, Repetitive throwing, 
hyperextension, fall on an outstretched hand , heavy lifting, and direct trauma have all been implicated .

Classification of Biceps tendon lesion and especially the SLAP lesions are classified into 4 basic and 6 further subtypes Type $I$ is characterized by fraying and a degenerative appearance of the superior labrum, Type II is a detachment of the superior labrum from the supraglenoid tubercle. Type III is a bucket handle tear that displaced into the joint while the biceps root remains stable, Type IV is a bucket handle tear where the tear propagates into the biceps tendon .

Because SLAP lesions are often associated with other abnormalities of the shoulder, the diagnosis is difficult. Nevertheless, a thorough history is necessary to detect the underlying lesion - patients complaining of this problem usually have vague shoulder pain exacerbated by activity with the arm overhead and possibly associated with popping, locking, and snapping if the unstable fragment is trapped between the humerus and glenoid surface. Instability symptoms may be present if the tear extends into the anterior ligament and labrum .

Although the history and physical examination findings of the different clinical tests described in literature, as will as improved imaging modalities such as magnetic resonance arthrography, are extremely important in understanding these lesions , the definitive diagnosis of Biceps tendon lesions is best made through diagnostic arthroscopy .

The treatment of lesions of the biceps tendon should begin with prolonged conservative care before any thought of operative intervention. If symptoms persist despite a well-managed supervised rehabilitation program, operative intervention may be indicated.

Treatment of these lesions is directed according to its type. Surgical management of SLAP lesions usually entails arthroscopic debridement or repair . in general, repairs are performed with either an absorbable tack or with anchor suture repair. Each approach has its unique set of advantages and disadvantages . current implant designs and advances in arthroscopic techniques have made suture repair technically less demanding , effectively compensating the relative case of absorbable tack insertion .

Type I lesions are treated with conservative debridement of the frayed labrum . Type II lesions are repaired using a single-anchor, double-suture technique . Type III lesions are treated by initial resection of the unstable bucket-handle labral fragment, followed by inspection of the biceps anchor attachment for stability. Type IV lesions are treated as type III lesions unless the biceps tendon split is severe. when more than approximately $30 \%$ of the tendon is included with the displaced labral tear one must consider either repairing the tendon, or releasing it and repairing the labrum as with a type II SLAP, or performing a biceps tenodesis . the decision depends on the age and activity level of the patient and the condition of the remainder of the biceps tendon .

\section{References}

[1] CL. Eakin, KJ. Faber, RJ. Hawkins, and WD. Hovis, Biceps tendon disorders in atheletes. Journal of the American Academy of Orthopaedic Surgeons, Vol.7, PP.300-310, 1999.

[2] CT Jr Vangsness, SS. Jorgenson, T. Watson, The origin of the long head of the biceps from the scapula and glenoid labrum: An anatomical study of 100 shoulders. Journal of Bone and Joint Surgery Br, Vol. 76, PP. 951954, 1994.

[3] JR. Andrews, Shoulder arthroscopy opening new pathways: An illustrated guide to shoulder arthroscopy. Smith and Nephew Dyonics inc,vol19,PP.133-140,1991.

[4] E. Itoi, DK. Kuechle, SR. Newman, Stabilizing function of the biceps in stable and unstable shoulders. Journal of Bone and Joint Surgery, Vol. 7J5, PP. 546 -550, 1993.

[5] CL Eakin, KJ Faber, RJ Hawkins, Biceps tendon disorders in atheletes. Journal of the American Academy of Orthopaedic Surgeons, Vol. 7, PP. 300-310, 1999.

[6] MJ Pagnani, XH Deng, RF Warren, Role of the long head of the biceps brachii in glenohumeral stability: A biomechanical study in cadavera. Journal of Shoulder and Elbow Surgery, Vol. 5, PP. 255-262, 1996.

[7] JH. Healey, S. Barton, P. Noble, Biomechanical evaluation of the origin of the long head of the biceps tendon. Arthroscopy, Vol. 17, PP.378-382, 2001.

[8] WZ Jr Burkhead, A. Michel, Arcand, The biceps tendon, in Rockwood CA Jr, Matsen FA III [eds]: The Shoulder. Philadelphia: WB Saunders, vol 3, PP.1009-1063, 2000.

[9] P. Slatis and K. Aalto, Medial dislocation of the tendon of the long head of the biceps brachii. Acta orthop. Scand, Vol. 50, PP. 73 77, 1979.

[10] P. Habermeyer and G. Walch, The biceps tendon and rotator cuff disease, in Burkhead WZ Jr [ed]: Rotator cuff disorders. Media, PA: Williams and Wilkins, Vol. 142,PP.199205, 1996.

[11] G. Walch, La pathologie de la longue portion du biceps. Conference d'enseignement de la SOFCOT, Paris,Vol.99,PP.121-125,1993.

[12] WZ Jr Burkhead, A. Michel, The biceps tendon, in Rockwood CA Jr, Matsen FA III [eds]: The Shoulder. Philadelphia: WB Saunders, vol 3, PP.1009-1063,2000.

[13] AS. Curtis, SJ. Snyder, Evaluation and treatment of biceps tendon pathology. Orthop Clin North Am, Vol. 24, PP. 33-43, 1993. 
[14] AS. Curtis, and SJ. Snyder, Evaluation and treatment of biceps tendon pathology. Orthop. Clin. North Am., Vol. 24, PP. 33-44, 1993.

[15] H. Lilleby Shoulder arthroscopy. Acta Orthop. Scand, Vol. 55, PP. 561-566, 1984.

[16] AM. Soliman, Arthroscopy as a diagnostic tool in shoulder disorders. M.D. Thesis, Kasr El-Aini, Faculty of MedicineVol.99,PP.99103,1995.

[17] PM. Ahrens, P. Boileau, The long head of biceps and associated tendinopathy. J Bone Joint Surg Br. Aug, Vol. 89, PP. 1001-9. 2007.

[18] EM. Mariani, RH. Cofield, LJ. Askew, Rupture of the long head of the biceps brachii: Surgical versus nonsurgical treatment. Clin. Orthop, Vol. 228, PP. 233-239, 1988.

[19] DA. Connell, HG. Potter, Noncontrast magnetic resonance imaging of superior labral lesions. 102 cases confirmed at arthroscopic surgery. Am J Sports Med, Vol. 27, PP. 208213, 1999.

[20] D. Altchek, RF. Warren, MJ. Skyhar, T-plasty modification of the Bankart procedure for multidirectional instability of the shoulder. Journal of Bone Joint Surgery Am, Vol.73, PP. 105-112, 1991.

[21] DJ. Friedman, JC. Dunn, LD. Higgins, Proximal biceps tendon: injuries and management. Sports Med Arthrosc. Sep, Vol. 16, PP. 162-9, 2008.

[22] WF. Bennett, Visualization of the anatomy of the rotator interval and bicipital sheath. Arthroscopy, Vol. 17, PP. 107-111,2001.

[23] S. S. Burkhart, C. D. Morgan and W. B. Kibler,"Shoulder Injuries in Overhead Athletes. The 'Dead Arm' Revisited." Clinics in Sports Medicine 191 [2000]: 125-158. National Center for Biotechnology Information. National Library of Medicine.Vol. 14 ,PP. 1065-1066,2008.

[24] CA. Churgay, Diagnosis and treatment of biceps tendinitis and tendinosis. Am Fam Physician. Sep, Vol. 1; 80, PP. 470-6. 2009. 